\title{
ESTUDO RADIOGRÁFICO DA RECONSTRUÇÃO DO LIGAMENTO CRUZADO ANTERIOR PELA VIA TRANSTIBIAL
}

\author{
RADIOLOGICAL STUDY OF RECONSTRUCTION OF THE ANTERIOR CRUCIATE \\ LIGAMENT BY TRANSTIBIAL APPROACH
}

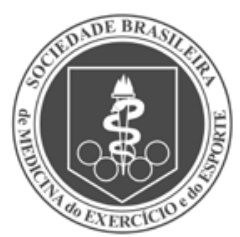

Artigo Original

\author{
ESTUDIO RADIOGRÁFICO DE LA RECONSTRUCCIÓN DEL LIGAMENTO \\ CRUZADO ANTERIOR POR TÉCNICA TRANSTIBIAL
}

Rafael de Souza Campos Fernandes ${ }^{1,2}$ (Médico)

Carlos Eduardo da Silveira Franciozi ${ }^{1,3}$ (Médico)

Ricardo de Souza Campos Fernandes² (Médico)

Marlon Alves Subtil de Oliveira' ${ }^{1}$ (Médico)

Laura Nascimento' (Médica)

Sheila Jean McNeill Ingham 1,3 (Médica)

Samir Salim Daher (Médico)

Jaques Waisberg² (Médico)

Rene Jorge Abdalla1,3 (Médico)

1. Instituto do Joelho. Hospital do Coração (HCor), São Paulo, SP, Brasil. 2. Instituto de Assistência Médica ao Servidor Público Estadual (IAMSPE), São Paulo, SP, Brasil. 3. Universidade Federal de São Paulo (Unifesp), São Paulo, SP, Brasil.

\section{Correspondência:}

Rua Rafael Danúncio Damiano, 277, Centro, Campos dos Goytacazes, RJ, Brasil, 28013-035.

rafa.scf@gmail.com

\section{RESUMO}

Introdução: A ruptura do ligamento cruzado anterior (LCA) é frequente devido às atividades esportivas, e sua reconstrução tem sofrido constantes modificações com o aprimoramento das técnicas cirúrgicas. Objetivo: Avaliar radiologicamente o posicionamento dos túneis tibial e femoral com o uso da técnica transtibial auxiliada pela pré-perfuração femoral. Método: Análise radiológica (AP e perfil), na quarta semana de pós-operatório de 98 pacientes, totalizando 100 casos de reconstrução do LCA. Três examinadores avaliaram o posicionamento dos túneis tibial e femoral pelos seguintes métodos: Scanlan, Staubli e Rauschning e Bernard. Resultados: O posicionamento do ângulo $\alpha$ (AP) foi de $64,13^{\circ}\left( \pm 4,29^{\circ}\right.$ ) e do ângulo $\beta$ (perfil), de $57,28^{\circ}$ $\left( \pm 4,41^{\circ}\right)$. A média de posicionamento tibial foi de $41,99 \%( \pm 5,14 \%)$. O enxerto do LCA foi inserido no côndilo lateral do fêmur e a média em porcentagem de posicionamento no quadrante verde foi de $62 \%$, no quadrante amarelo, de $37 \%$ e de $1 \%$ no vermelho. Conclusões: A técnica transtibial de reconstrução do LCA auxiliada pela pré-perfuração femoral proporciona o posicionamento anatômico do enxerto na grande maioria dos casos, conforme comprovação radiológica.

Palavras-chave: ligamento cruzado anterior, tíbia, joelho, reconstrução.

\begin{abstract}
Introduction: The rupture of the anterior cruciate ligament (ACL) is often due to sports activities and its reconstruction methods have undergone constant changes due to improvements in the surgical techniques. Objective: To evaluate the radiological placement of the tibial and femoral tunnels using the transtibial technique assisted by the femoral pre-drilling. Method: Radiological analysis (AP and lateral), at 4 weeks postoperatively in 98 patients, totaling 100 cases of $A C L$ reconstruction. Three examiners evaluated the placement of the tibial and femoral tunnels. Methods for assessing the positioning of the tunnels were: Scanlan, Staubli and Rauschning and Bernard. Results: The a angle (AP) was $64.13^{\circ}\left( \pm 4.29^{\circ}\right)$ and the $\beta$ angle (lateral) was $57.28^{\circ}\left( \pm 4.41^{\circ}\right)$. The mean tibial positioning was $41.99 \%$ ( $\pm 5.14 \%)$. The ACL graft was inserted into the lateral condyle of the femur and the average percentage of radiographic positioning in the green quadrant was $62 \%$, the yellow quadrant, $37 \%$ and $1 \%$ in the red quadrant. Conclusions: The transtibial technique for ACL reconstruction, assisted by the femoral pre-drilling provides the anatomical position of the graft in the majority of the cases, as radiological evidence.
\end{abstract}

Keywords: anterior cruciate ligament, tibia, knee, reconstruction.

\section{RESUMEN}

Introducción: La ruptura del ligamento cruzado anterior (LCA) es frecuente debido a las actividades deportivas, y su reconstrucción ha sufrido constantes modificaciones con la mejora de las técnicas quirúrgicas. Objetivo: Evaluar radiográficamente el posicionamiento de los túneles tibial y femoral con el uso de la técnica transtibial auxiliada por la pre-perforación femoral. Método: Análisis radiográfico (AP y perfil), en la $4^{a}$ semana de postoperatorio de 98 pacientes, totalizando 100 casos de reconstrucción del LCA. Tres examinadores evaluaron el posicionamiento de los túneles tibial y femoral por los siguientes métodos: Scanlan, Staubli y Rauschning y Bernard. Resultados: El posicionamiento del ángulo a (AP) fue de $64,1^{\circ}\left( \pm 4,29^{\circ}\right)$ y del ángulo $\beta$ (perfil), de $57,28^{\circ}\left( \pm 4,41^{\circ}\right)$. El promedio de posicionamiento tibial fue de $41,99 \%$ ( $\pm 5,14 \%)$. El injerto del $L C A$ fue colocado en el cóndilo lateral del fémur y el promedio en porcentaje de posicionamiento en el cuadrante verde fue de $62 \%$, en el cuadrante amarillo de $37 \%$ y de $1 \%$ en el rojo. Conclusiones: La técnica transtibial de reconstrucción del LCA auxiliada por la pre-perforación femoral proporciona el posicionamiento anatómico del injerto en la gran mayoría de los casos, de acuerdo a la comprobación radiográfica.

Palabras clave: ligamento cruzado anterior, tibia, rodilla, reconstrucción. 


\section{INTRODUÇÃO}

A lesão do ligamento cruzado anterior (LCA), além de produzir episódios de falseio, altera a mecânica articular. A sua ruptura é uma das mais freqüentes lesões da articulação do joelho na prática esportiva e geralmente exige abordagem cirúrgica. A reconstrução do LCA é um dos procedimentos ortopédicos que mais tem recebido atenção nos últimos tempos levando a uma constante procura de aprimoramento das técnicas e melhoria dos resultados'.

Inúmeros procedimentos cirúrgicos têm sido descritos com o objetivo de prevenir a instabilidade sintomática e o processo degenerativo articular prematuro. Uma das grandes alteraç̃es no procedimento cirúrgico foi a reintrodução da cirurgia anatômica do LCA em que o enxerto é colocado na área de inserção nativa do LCA'. Na técnica cirúrgica anterior, dita isométrica, em que o túnel femoral é geralmente realizado de maneira transtibial, a origem do túnel femoral costuma ficar alta em comparação à origem anatômica do LCA, resultando em uma posição verticalizada do enxerto nos aspectos sagital e coronal ${ }^{2-5}$. Um enxerto verticalizado pode resistir à translação anterior da tíbia, mas não aos movimentos de rotação do joelho ${ }^{6}$. A colocação correta do túnel é de fundamental importância para uma boa evolução clínica após a reconstrução do $L_{C A} A^{7}$. A reconstrução anatômica do LCA é o ponto chave para o sucesso da cirurgia e a colocação não anatômica do enxerto pode resultar em uma cinemática anormal do joelho levando ao impacto do enxerto ou estresse do mesmo $0^{7,8}$.

Com a introdução do conceito de cirurgia anatômica houve também a introdução do uso de três portais para facilitar a visualização da parede medial do côndilo femoral lateral9. Com isso, a técnica transportal para a perfuração do fêmur facilita a colocação anatômica do enxerto ${ }^{24-6}$. No entanto, muitos cirurgiões ainda sentem-se confortáveis com o uso da técnica transtibial para a perfuração do fêmur, o que, em tese, resultaria em uma colocação não-anatômica do enxerto, em uma posição mais alta na parede do côndilo lateral ${ }^{2,4-8}$. Este trabalho tem como objetivo avaliar o posicionamento dos túneis tibial e femoral, através da radiografia simples anteroposterior e lateral, pela técnica transtibial auxiliada pela pré-perfuração femoral na reconstrução da banda simples do LCA.

\section{MÉTODOS}

Este projeto foi aprovado pela Comissão de Ética em Pesquisa da nossa instituição, de acordo com a Resolução 196/96 do Conselho Nacional de Saúde (Diretrizes e Normas Regulamentadoras de Pesquisa Envolvendo Seres Humanos). Este é um estudo retrospectivo de análise de uma série de casos.

Foram avaliadas 100 radiografias (98 pacientes, dois casos bilaterais) de joelhos de pacientes submetidos à reconstrução do LCA com a técnica transtibial. O autoenxerto foi utilizado em todos os casos. Foram excluídos pacientes com lesões multiligamentares e os que apresentavam radiografias fora do padrão proposto.

\section{Técnica cirúrgica}

Antes da cirurgia, todos os pacientes foram examinados sob anestesia para confirmar o diagnóstico pré-operatório definido por exames físico e de imagem. Para o procedimento cirúrgico, os pacientes foram posicionados em decúbito dorsal, com manguito pneumático insuflado a 350 $\mathrm{mmHg}$. Todos foram submetidos à mesma técnica cirúrgica e operados pelo mesmo cirurgião, com uso de tendões flexores ou patelar para a reconstrução do LCA com banda simples.

Após a exploração articular, identificam-se as inserções femoral e tibial com o artroscópio inserido no portal anterolateral. No fêmur, utiliza-se como parâmetros a crista intercondilar lateral e a crista bifurcada lateral, além do próprio coto do LCA, quando presente ${ }^{10}$. Na tíbia, utiliza-se como parâmetros a borda posterior do corno anterior do menisco lateral, a espinha tibial medial e o próprio coto do LCA, quando presente ${ }^{10}$. No fêmur, após sulcoplastia econômica, quando necessária para visualização adequada, dividiu-se o côndilo lateral segundo orientação proximal-distal em proximal ou profundo e distal ou superficial e segundo orientação anteroposterior em anterior ou alto ou superior e posterior ou baixo ou inferior ${ }^{10}$. Segundo essas orientações, marca-se com ice-pick, no terço inferior segundo orientação anteroposterior e no ponto médio segundo orientação proximal distal um ponto que corresponde à inserção femoral nativa do LCA ${ }^{10}$ (figura 1a). Introduz-se o fio guia flexível pelo portal anteromedial (transportal) no ponto previamente marcado com o ice-pick e antes perfura-se $1 \mathrm{~cm}$ no fêmur com a broca flexível guiada pelo fio flexível (Clancy Anatomic Cruciate Guide/ Flexible Drill System; Smith \& Nephew, Andover, MA) (figuras $1 \mathrm{~b}, c)$. A pré-perfuração femoral com apenas $1 \mathrm{~cm}$ de profundidade visa criar o ponto adequado para a introdução do fio e da broca rígida de maneira transtibial, mantendo com isso o posicionamento anatômico da inserção femoral. Na tíbia, o guia do LCA, que orienta o ponto de saída do fio-guia, é posicionado de forma que o fio localize-se levemente anterior à borda posterior do corno anterior do menisco lateral e levemente medial à linha média da inserção tibial do LCA ${ }^{10}$ (figura 1d). Perfura-se a tíbia com broca canulada de diâmetro adequado ao enxerto orientada pelo fio guia. $\mathrm{O}$ joelho é fletido a $110^{\circ}$ e introduz-se um fio guia rígido, pela via transtibial, no ponto femoral pré-perfurado (figura 1e). Ainda pela via transtibial, introduz-se a broca rígida de diâmetro adequado ao endobutton (Smith \& Nephew, Andover, MA) e o enxerto é passado com fio guia rígido (figura 1f).

\section{Avaliação Radiográfica}

Radiografias da quarta semana de pós-operatório foram avaliadas. As radiografias foram realizadas no mesmo aparelho $\left(\right.$ Philips $\left.^{\circledR}\right)$, com a mesma técnica e foram realizadas duas incidências: Incidência 1 (AP convencional): radiografia anteroposterior do joelho em extensão com carga em apoio bipodal. A distância tubo-filme foi de 1 metro e os raios $X$ centrados no pólo inferior da patela, sendo o tamanho do filme 30x35cm; incidência 2 (Perfil): radiografia em perfil do joelho com flexão de $10^{\circ} \mathrm{em}$ posição ortostática com a distância tubo-filme padronizada em 1 metro, tendo o mesmo tamanho de filme. Todas as radiografias foram avaliadas por três ortopedistas especialistas em Cirurgia do Joelho.

A posição do túnel femoral foi avaliada de acordo com o método dos quadrantes descrito por Bernard et al. ${ }^{11}$ e modificado por Sommer et al. ${ }^{12}$ (figura 2a). As angulações a (AP) e $\beta$ (perfil) foram avaliadas utilizando-se o método de Scanlan et al.13 (figuras 2 b e c). A posição do túnel tibial foi avaliada pelo método de Staubli e Rauschning ${ }^{14}$ (figura 2d).

Em resumo, na radiografia AP, uma reta tangencial à superfície articular foi traçada e um ângulo criado, entre a posição do centro do túnel tibial no plano coronal e a inserção no côndilo femoral no limite inferior do túnel femoral (figura 2b). Na radiografia em perfil, uma linha tangenciando a superfície articular do platô tibial foi traçada e o ângulo que esta forma com o ponto de saída do enxerto do túnel tibial e o ponto da inserção no côndilo femoral constitui o ângulo $\beta$ (figura 2c). Além disto, na radiografia de perfil traçamos uma reta tangenciando a linha de Blumensaat e uma outra perpendicular a essa no ponto de encontro com a cortical femoral do côndilo lateral definindo os 4 quadrantes femorais: verde (mais posterior), amarelo, vermelho e azul (mais anterior),(figura 2a). Na radiografia de perfil da tíbia traçamos uma linha através da superfície articular tibial que vai da cortical anterior, ponto 0\%, até a cortical posterior, ponto 100\% (figura 2d). 

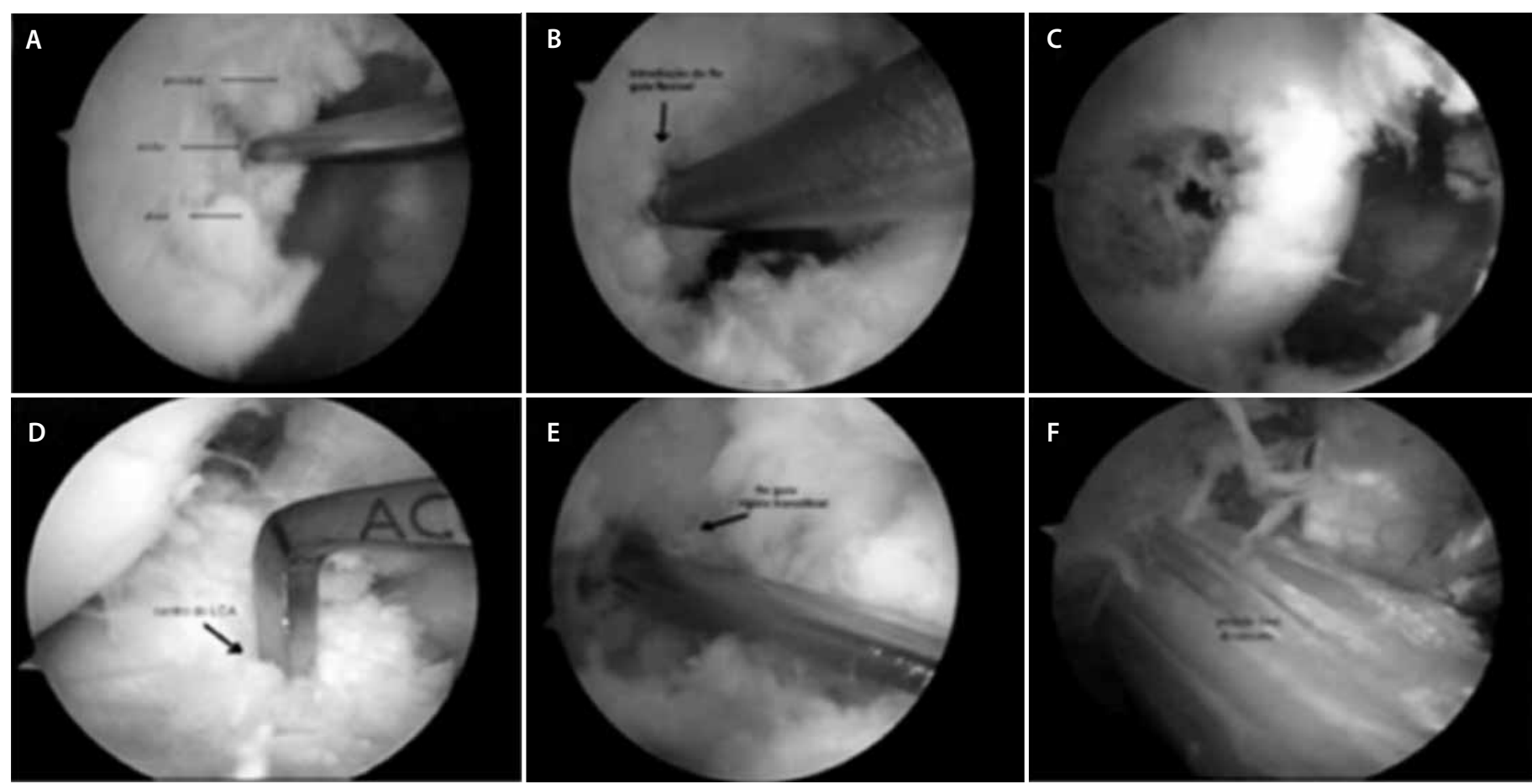

Figura 1. A) Marcação com ice-picking após a trocleoplastia; B) Local do côndilo femoral lateral onde foi realizada a perfuração do túnel; C) Relação com a cortical posterior do côndilo; D) Posicionamento do guia tibial; E) Passagem do fio guia; F) Posição final do enxerto do LCA.

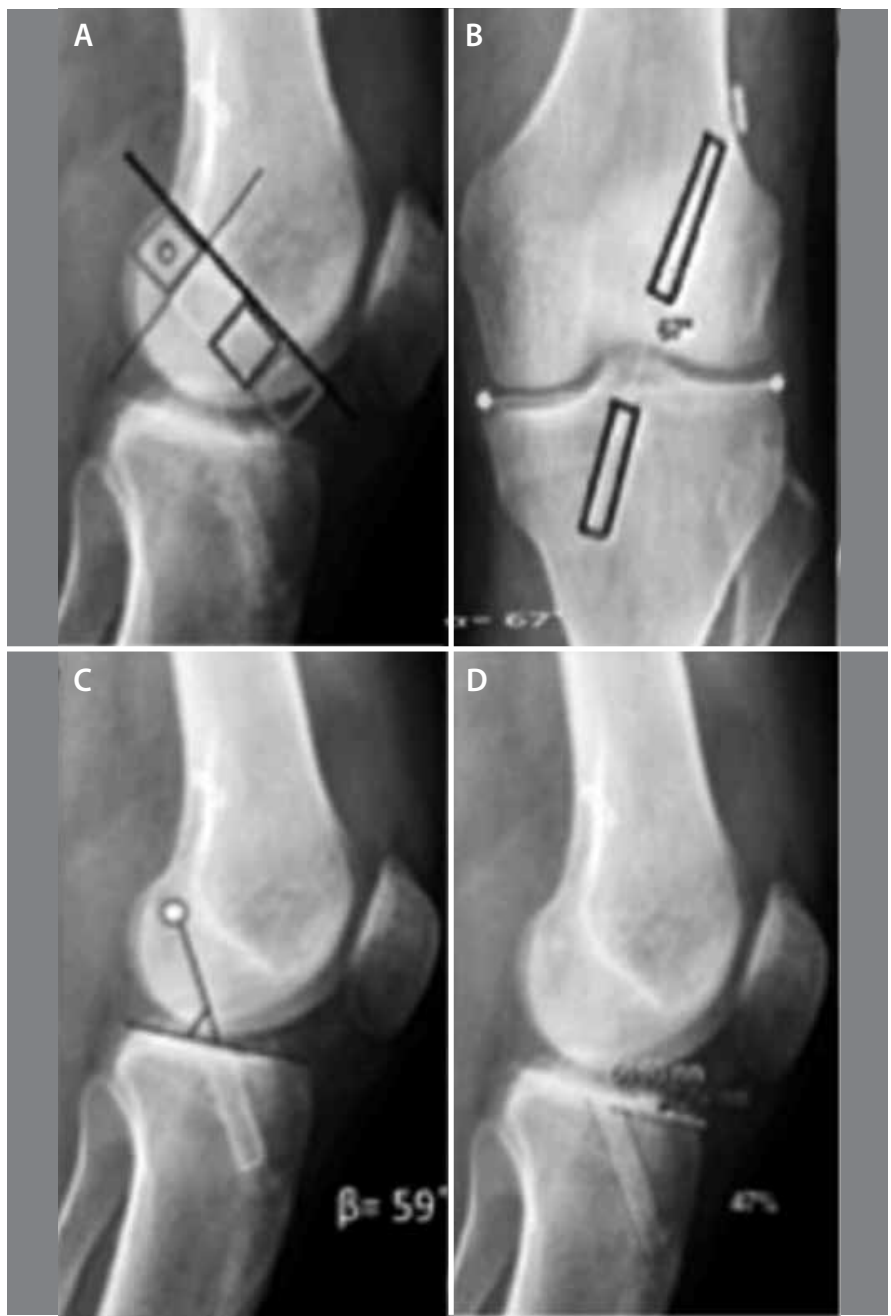

Figura 2. A) Radiografia perfil mostrando a marcação de um quadrante femoral (verde); B) Radiografia AP mostrando o ângulo a; C) Radiografia perfil mostrando o ângulo $\beta ;$ D) Radiografia perfil mostrando a marcação do túnel tibial pelo método de Staubli e Rauschning ${ }^{13}$

\section{Análise estatística}

Os valores do ângulo anteroposterior (a), de perfil ( $\beta$ ) e a porcentagem da inserção no platô tibial foram descritos para cada avaliador e para média dos valores dos três avaliadores com uso de medidas resumo (média, desvio padrão, mediana, mínimo, máximo e intervalo com 95\% de confiança para a média) para verificar se estes parâmetros estavam em consonância com seus padrões de literatura.

A avaliação do quadrante de apresentação no côndilo femoral foi descrita com uso de frequências absolutas e relativas e foi criado um resultado final dos três avaliadores em que prevalecesse a resposta da maioria. No caso de discordância dos três avaliadores foi atribuído quadrante amarelo para o resultado conjunto dos três, sendo este resultado final descrito da mesma maneira.

Para avaliar a concordância entre as mensurações angulares e de porcentagem entre os três avaliadores foi calculado o coeficiente de correlação intra-classe individual e para a média das três avaliações com os respectivos intervalos de 95\% de confiança e apresentando os gráficos de Bland-Altman para verificar qualquer tendência entre as discordâncias nas mensurações.

Para avaliação da reprodutibilidade da mensuração do quadrante de apresentação côndilo-femoral entre os três avaliadores foi calculado o coeficiente Kappa ponderado total com os respectivos intervalos com 95\% de confiança.

\section{RESULTADOS}

Foram avaliadas 100 radiografias de 98 pacientes (84 do sexo masculino - 85,7\%). A média de idade foi de 34 anos, variando de 15 a 63 anos, sendo realizadas 69 reconstruções com enxerto de tendões flexores e 31 com tendão patelar.

Os resultados referentes à angulação dos túneis tibial e femoral e da porcentagem tibial na reconstrução transtibial de 100 casos são demonstrados na tabela 1. Tivemos como média os seguintes valores para posicionamento do ângulo a $(\mathrm{AP})=64,13^{\circ}\left( \pm 4,29^{\circ}\right)$ e ângulo $\beta(\mathrm{P})=$ $57,28^{\circ}\left( \pm 4,41^{\circ}\right)$. Já a análise das radiografias da tíbia mostra que a média de posicionamento do túnel tibial foi de $41,99 \%$ ( $\pm 5,14 \%)$. A tabela 2 mostra a concordância entre os avaliadores. A tabela 3 mostra a posição em que o enxerto do LCA foi colocado no côndilo lateral femoral. 
Tabela 1. Angulações a, $\beta$ e a porcentagem tibial analisada por 3 avaliadores cegos.

\begin{tabular}{|c|c|c|c|c|c|c|c|c|c|}
\hline \multirow{2}{*}{ Variável } & \multirow{2}{*}{ Avaliador } & \multirow{2}{*}{ Média } & \multirow{2}{*}{$\mathrm{DP}$} & \multirow{2}{*}{ Mediana } & \multirow{2}{*}{ Mínimo } & \multirow{2}{*}{ Máximo } & \multicolumn{2}{|c|}{ IC (95\%) } & \multirow{2}{*}{$\mathrm{N}$} \\
\hline & & & & & & & Inferior & Superior & \\
\hline \multirow{4}{*}{$\mathrm{AP}(\mathrm{a})$} & 1 & 65,11 & 4,65 & 65,00 & 53 & 74 & 64,20 & 66,02 & 100 \\
\hline & 2 & 63,43 & 4,84 & 63,00 & 50 & 74 & 62,48 & 64,38 & 100 \\
\hline & 3 & 63,85 & 5,25 & 63,00 & 50 & 78 & 62,82 & 64,88 & 100 \\
\hline & Média & 64,13 & 4,29 & 63,67 & 51 & 75,0 & 63,29 & 64,97 & 100 \\
\hline \multirow{4}{*}{$P(\beta)$} & 1 & 58,61 & 4,88 & 58,50 & 48 & 70 & 57,65 & 59,57 & 100 \\
\hline & 2 & 56,70 & 5,50 & 57,00 & 44 & 70 & 55,62 & 57,78 & 100 \\
\hline & 3 & 56,53 & 5,55 & 57,00 & 43 & 67 & 55,44 & 57,62 & 100 \\
\hline & Média & 57,28 & 4,41 & 56,67 & 46 & 66,7 & 56,42 & 58,14 & 100 \\
\hline \multirow{4}{*}{$\%$ t. } & 1 & 40,49 & 6,20 & 41,00 & 21 & 58 & 39,28 & 41,70 & 100 \\
\hline & 2 & 42,19 & 5,94 & 42,00 & 32 & 61 & 41,03 & 43,35 & 100 \\
\hline & 3 & 43,29 & 5,96 & 43,00 & 32 & 61 & 42,12 & 44,46 & 100 \\
\hline & Média & 41,99 & 5,14 & 42,17 & 33,0 & 60 & 40,98 & 43,00 & 100 \\
\hline
\end{tabular}

Tabela 2. Avaliação da concordância entre as mensurações angulares e de porcentagem entre os três avaliadores.

\begin{tabular}{|c|c|c|c|c|}
\hline \multirow{2}{*}{ Variável } & \multirow{2}{*}{ Reprodutibilidade } & \multirow{2}{*}{$\begin{array}{l}\text { Correlação } \\
\text { Intraclasse }\end{array}$} & \multicolumn{2}{|c|}{ IC (95\%) } \\
\hline & & & Inferior & Superior \\
\hline \multirow{2}{*}{$\mathrm{AP}(\mathrm{a})$} & Induvidual & 0,623 & 0,516 & 0,716 \\
\hline & Média & 0,832 & 0,762 & 0,883 \\
\hline \multirow{2}{*}{$P(\beta)$} & Induvidual & 0,509 & 0,387 & 0,620 \\
\hline & Média & 0,756 & 0,655 & 0,831 \\
\hline \multirow{2}{*}{$\% \mathrm{t}}$. & Induvidual & 0,561 & 0,438 & 0,669 \\
\hline & Média & 0,793 & 0,701 & 0,858 \\
\hline Côndilo-Femoral* & & 0,527 & 0,340 & 0,715 \\
\hline
\end{tabular}

Tabela 3. Porcentagem do local de colocação do enxerto do LCA no túnel femoral, na radiografia de perfil do joelho.

\begin{tabular}{c|c|c|c}
\hline Avaliador & Côndilo-Femoral & N & $\%$ \\
\hline \multirow{4}{*}{ Avaliador 1 } & Verde & 57 & 57,0 \\
\cline { 2 - 4 } & Amarelo & 40 & 40,0 \\
\cline { 2 - 4 } & Vermelho & 3 & 3,0 \\
\hline \multirow{4}{*}{ Avaliador 2 } & Verde & 64 & 64,0 \\
\cline { 2 - 4 } & Amarelo & 35 & 35,0 \\
\cline { 2 - 4 } & Vermelho & 1 & 1,0 \\
\hline \multirow{3}{*}{ Avaliador 3 } & Verde & 61 & 61,0 \\
\cline { 2 - 4 } & Amarelo & 38 & 38,0 \\
\cline { 2 - 4 } & Vermelho & 1 & 1,0 \\
\hline \multirow{2}{*}{$\begin{array}{c}\text { Resultado final dos três } \\
\text { avaliadores conjuntamente }\end{array}$} & Verde & 62 & 62,0 \\
\cline { 2 - 4 } & Amarelo & 37 & 37,0 \\
\cline { 2 - 4 } & Vermelho & 1 & 1,0 \\
\hline \multicolumn{2}{c}{ Total } & 100 & 100 \\
\hline
\end{tabular}

\section{DISCUSSÃO}

A técnica convencional transtibial para a reconstrução do LCA foi muito utilizada desde sua popularização sobre a técnica inicial de "duas incisões" onde o túnel femoral era realizado de fora para dentro. Com isso a técnica transtibial, reconhecida como "uma incisão", tornou-se a mais frequente por evitar uma incisão lateral no fêmur, diminuir o tempo cirúrgico e possivelmente a morbidade ${ }^{8}$. Apesar destas vantagens iniciais, notou-se que o posicionamento obtido não era anatômico e que isso poderia acarretar maior instabilidade ${ }^{1-8}$.

A maior crítica às reconstruções transtibiais é a alta frequência de posicionamentos não-anatômicos do enxerto, principalmente no fêmur ${ }^{2,4-9}$. Nesse contexto, faz-se mister uma modificação técnica que permita a resolução do problema. A realização da pré-perfuração femoral transportal permitiu o posicionamento anatômico do enxerto utilizando o método transtibial na maioria dos casos, segundo os métodos radiológicos utilizados.

Diversos métodos foram propostos para avaliar radiograficamente a localização do enxerto através dos túneis femoral e tibial 1,11-14. Dentre eles, destacam-se o de Sommer et al. ${ }^{12}$, Staubli et al. ${ }^{14}$ e Scanlan et $a l^{13}{ }^{13}$, conforme já mencionado em outros estudos ${ }^{1,7}$. Bernard et al. ${ }^{11}$ descreveram que o centro da inserção femoral na radiografia de perfil apresenta-se aproximadamente entre os dois quadrantes mais posteriores (o verde e amarelo), o que é confirmado pelos nossos dados (62\% no quadrante verde e 37\% no amarelo). Na tíbia, Staubli e Rauschning ${ }^{14}$ encontraram a inserção do LCA na posição entre 25 e $62 \%$ do diâmetro sagital e recomendaram a colocação do túnel para o enxerto em 43\%. Obtivemos uma média de 41,99\% ( \pm 5,14\%), bem próxima do valor proposto. Já Scanlan et al. ${ }^{13}$, mostraram que o LCA nativo na incidência $A P$ variou entre $58^{\circ}$ e $75^{\circ}$ e no Perfil entre $45^{\circ}$ e $67^{\circ}$. Nossos resultados mostram uma média para o ângulo a (AP) de $64,13^{\circ} \pm 4,29^{\circ}$ e para o ângulo $\beta(P)$ de $57,28^{\circ} \pm 4,41^{\circ}$, também situando-se dentro dos valores de normalidade para o posicionamento anatômico. Mostra-se que, com o uso da pré-perfuração femoral, é possível utilizar-se a técnica transtibial e obter um posicionamento anatômico, normalmente não obtido com a técnica clássica7,8. Isto pode ser importante, pois o túnel femoral realizado de maneira transportal costuma ter comprimento inferior ao túnel femoral realizado de maneira transtibial o que pode comprometer a quantidade de enxerto a ser incorporado de acordo com o método de fixação utilizado. Na técnica transtibial auxiliada pela pré-perfuração femoral incorpora-se a vantagem dos métodos transportal e transtibial, visto que consegue-se um posicionamento anatômico do enxerto através da pré-perfuração transportal e um túnel femoral mais longo através da sua finalização transtibial. Um erro freqüente da técnica transtibial é a colocação excessivamente posterior do túnel no lado tibial, associada 
a uma localização femoral muito alta e/ou anterior na parede medial do côndilo femoral lateral, possibilitando o impacto do enxerto sobre o ligamento cruzado posterior e um posicionamento vertical. Heming et al. ${ }^{15}$ afirmam que para atingir a trajetória ideal transtibial é necessário um ponto de partida tibial a milímetros da borda do platô medial, o que implica que as posições mais distais de partida possam ser incapazes de prover a inserção femoral anatômica pela via transtibial clássica, uma limitação superada através da técnica transtibial auxiliada pela pré-perfuração femoral demonstrada no corrente estudo.

\section{CONCLUSÃO}

A técnica transtibial de reconstrução do LCA auxiliada pela pré-perfuração femoral proporciona o posicionamento anatômico do enxerto na grande maioria dos casos, conforme demonstrado pela avaliação radiológica realizada no presente estudo.

Os autores Carlos Eduardo da Silveira Franciozi e Rene Jorge Abdalla declaram que são consultores da PCE.

\section{REFERÊNCIAS}

1. Piefer JW, Pflugner TR, Hwang MD, Lubowitz JH. Anterior cruciate ligament femoral footprint anatomy: systematic review of the 21st century literature. Arthroscopy. 2012;28(6):872-81.

2. Arnold MP, Kooloos J, van Kampen A. Single-incision technique misses the anatomical femoral anterior cruciate ligament insertion: a cadaver study. Knee Surg Sports Traumatol Arthrosc. 2001;9(4):194-9.

3. Morgan CD, Kalmam VR, Grawl DM. Isometry testing for anterior cruciate ligament reconstruction revisited. Arthroscopy. 1995;11(6):647-59.

4. Kopf S, Forsythe B, Wong AK, Tashman S, Anderst W, Irrgang JJ, et al. Nonanatomic tunnel position in traditional transtibial single-bundle anterior cruciate ligament reconstruction evaluated by three-dimensional computed tomography. J Bone Joint Surg Am. 2010;92(6):1427-31.

5. Takeda Y, Iwame T, Takasago T, Kondo K, Goto T, Fujii K, et al. Comparison of tunnel orientation between transtibial and anteromedial portal techniques for anatomic double-bundle anterior cruciate ligament reconstruction using3-dimensional computed tomography. Arthroscopy. 2013;29(2):195-204.

6. Bedi A, Musahl V, Steuber V, Kendoff D, Choi D, Allen AA, et al. Transtibial versus anteromedial portal reaming in anterior cruciate ligament reconstruction: an anatomic and biomechanical evaluation of surgical technique.Arthroscopy. 2011;27(3):380-90.

7. Marchant BG, Noyes FR, Barber-Westin SD, Fleckenstein C. Prevalence of nonanatomical graft placement in a series of failed anterior cruciate ligament reconstructions. Am J Sports Med. 2010;38(10):1987-96.

8. Alentorn-Geli E, Samitier G, Alvarez P, Steinbacher G, Cugat R. Anteromedial portal versus transtibial

drilling techniques in ACL reconstruction: a blinded cross-sectional study at two- to five-year follow -up. Int Orthop. 2010;34(5):747-54

9. Bottoni CR, Rooney RC, Harpstrite JK, Kan DM. Ensuring accurate femoral guide pin placement in anterior cruciate ligament reconstruction. Am J Orthop (Belle Mead NJ). 1998;27(11):764-6.

10. Brown CH Jr, Spalding T, Robb C. Medial portal technique for single-bundle anatomical Anterior Cruciate Ligament (ACL) reconstruction. Int Orthop. 2013;37(2):253-69.

11. Bernard $M$, Hertel $P$, Hornung $H$, Cierpinski T. Femoral insertion of the ACL. Radiographic quadrant method. Am J Knee Surg. 1997;10(1):14-21.

12. Sommer C, Friederich NF, Müller W. Improperly placed anterior cruciate ligament grafts: correlation between radiological parameters and clinical results. Knee Surg Sports Traumatol Arthrosc. 2000;8(4):207-13.

13. Scanlan SF, Blazek K, Chaudhari AM, Safran MR, Andriacchi TP. Graft orientation influences the knee flexion moment during walking in patients with anterior cruciate ligament reconstruction. Am J Sports Med. 2009;37(11):2173-8.

14. Stäubli HU, Rauschning W. Tibial attachment area of the anterior cruciate ligament in the extended knee position. Anatomy and cryosections in vitro complemented by magnetic resonance arthrography in vivo. Knee Surg Sports Traumatol Arthrosc. 1994;2(3):138-46.

15. Heming JF, Rand J, Steiner ME. Anatomical limitations of transtibial drilling in anterior cruciate ligament reconstruction. Am J Sports Med. 2007;35(10):1708-15 